\title{
Low-dose Radiation Effects to Humans and the Importance of Eating Wisely
}

\author{
Louis Pasteur Center for Medical Research, Kazuko Uno
}

More than three years have passed since the Fukushima Daiichi Nuclear Power Plant Accident caused by the Great East Japan earthquake on March 11, 2011. Still, there are more than 120,000 evacuees who were forced to take refuge elsewhere, in and outside the prefecture. Immediately after the accident, residents were severely anxious about the health effects of low-dose radiation, especially on children. Some people are relieved that three and a half years later, there are no significant effects despite their original concerns. However, radiation is still a significant emotional burden on residents in Fukushima prefecture as many people are still uneasy about the possible long-term effects. This paper is based on a presentation "The effects of low-dose radiation on humans and the importance of eating wisely," that was geared towards women, and presented at the Atomic Energy Society of Japan Symposium. Although my expertise is in immunology and not radiology, being deeply involved in the radiation and other dire issues facing Fukushima from March 2011, has shaped my awareness of the complexities involved. Additionally, actively doing outreach activities with evacuees and other residents in Fukushima several times a month for the past three years combined with specialized knowledge has allowed the author to for focus on relevant subjects that other researchers do not touch upon. Some information in this article may overlap those from a previous article "My Reasons for Writing the Book, "Overcoming Low-Dose Radiation Contamination" which appeared in this journal last year.

\section{Importance of Thinking Scientifically}

\section{1, What is the Most Influential Factor for Cancer Risk? Does Fear Reduce Immunity and Increase the Risk of Cancer More Than Exposure to Low-Dose Radiation?}

From March 11, 2011, until now, I have been explaining the effects of low-dose radiation to concern citizens in Fukushima. If the main concern in regard to low dose radiation is the risk of cancer, then my prior research has led me to believe that the introduction of a lifestyle that improves the immune function might be effective. 
In my studies, I measured the immune function of several thousand of cancer patients, patients inflicted with diseases such as hepatitis and diabetes and healthy individuals. The immune function in the sample groups was measured using several parameters including interferon (IFN) -production ability. IFN production ability was measured by quantifying the IFN produced in patients blood sample against a viral infection simulated in a test tube ${ }^{1)}$. The activity of natural killer cells against cancer cells that they attack cancer cells appeared in the body almost every day was also measured. I also developed a method to assess whether or not an immune response occurred in a cancerous site in a patient, and found that the prognosis was better in patients with activated T cells and that the performance status (PS) and quality of life (QOL) of the patients were more important for the immune function (although the size and stage of the cancer surely influenced the same) ${ }^{2}$. PS is an approximation of cancer patients' general well-being and physical activity level in their daily life. A healthy and active cancer patient would be rated (PS0), up and about more than $50 \%$ of the day (PS2), and bedridden (PS4). These results became the contents of presentations I gave lecture exclusively to cancer patients; these presentations were also posted on the home page of "NPO Einstein," a volunteer group of scientists to which I belong just at the end of March, 2011.

Based on the aforementioned data, and other exhaustive studies done on radiation and the onset of cancer, I personally, even in 2011, did not believe that cancer risk would increase by a significant amount due to the radiation levels in Fukushima (except in the evacuated area where the radiation dosage was especially high). Rather, I believed that a decrease in immune function caused by people's excessive concern about the effects of the radiation and the stress associated with living as evacuees might increase their cancer risk. About a month after the accident I also become concerned about the high number of children in metropolitan areas who left vegetables from their school lunches uneaten because they were worried about radioactive contamination in the food. Hearing this, I have consistently warned that a lack of vegetables also increases cancer risk. I still talk about the relationship between cancer and immune function based on past studies, and try to introduce the foods and lifestyle that prevent cancer.

\section{Effects of Radiation and Reactive Oxygen Spaces}

So-called oxygen radical (reactive oxygen species), such as the superoxide and hydroxyl radical produced when radical hits water in the body, indirectly damaging a gene is more common than damage from radiation hitting a gene directly. This issue is an extension of my research specialty, and so I could play a role in explaining these effects. Recently, it has been shown that the effect of oxygen radical, namely those of oxidative stress, has been substantial in various so-called adult diseases including cancer. In the field of longevity and anti-aging medicine, oxidative stress is considered the chief culprit that causes rust in the body and has been found to be responsible for many age-related diseases, such as arteriosclerosis, myocardial infarction, Alzheimer's, cancer, diabetes, gastric ulcers, and cataracts.

Aging and inflammation was a topic of particular interest to me even before the Daiichi Accident. I believe that the accentuation of a particularly weak chronic inflammation was related to various diseases and many so-called adult diseases ${ }^{3)}$. In fact, it has been proven that IL-6, an inflammatory cytokine produced in the presence chronic inflammation, tends to increase as people age. Meanwhile, it was also found that this IL-6 had a dose-dependent, upward trend of exposure in atomic bomb survivors ${ }^{4}$.

Recent research has been probing into diseases other than cancer that may be influenced by radiation. Data accumulated on the aging population of atomic bomb survivors, show that the risk of circulatory diseases in particular, increases under the effects of radiation. The life 
expectancy survey conducted on the survivors of the atomic bombing in Hiroshima and Nagasaki, revealed that the effects of radiation are more increasingly apparent as the follow-up period increases. In other words, in the study done from 1950 through 1965, a dose dependency was not observed at 1 Gy or less; while in the study conducted from 1966 through 2003, a dose dependent relationship was observed between radiation and the severity of circulatory diseases even for survivors who were exposed to lower doses of radiation ${ }^{5)}$.

The excess relative risk of circulatory disease per $1 \mathrm{~Gy}$ of radiation exposure was 0.11 , which was not much larger than 0.47 for solid carcinoma. However, when we examined the cause of death of 50,620 people, who died between 1950 and 2003, the number of people who died of all types of solid carcinoma such as stomach cancer, colon cancer, and breast cancer (whole solid carcinoma), was 10,929 , while the number of people who died of circulatory system disease was 19,054 . This is approximately two times more than the total deaths from cancer. This excess dose dependency is still evident even at lower doses as the study period gets longer.

Recently, it has also been found that oxygen radicals which causes various diseases, is produced by not only ionizing radiation but also by smoking, obesity, various environmental factors, and repeated infections (Figure 1).

Reactive oxygen is not always bad for living organisms, on the contrary, it is essential. Many aerobic organisms produce reactive oxygen in a metabolic process using oxygen in the mitochondria to obtain the energy necessary to survive. This activated oxygen damages cells and may lead to cancer and other diseases including so-called adult diseases. On the other hand, living organisms also use oxygen radicals for sterilization and detoxification, and terrestrial organisms in particular, have developed a system that erases any damage caused by activated oxygen.

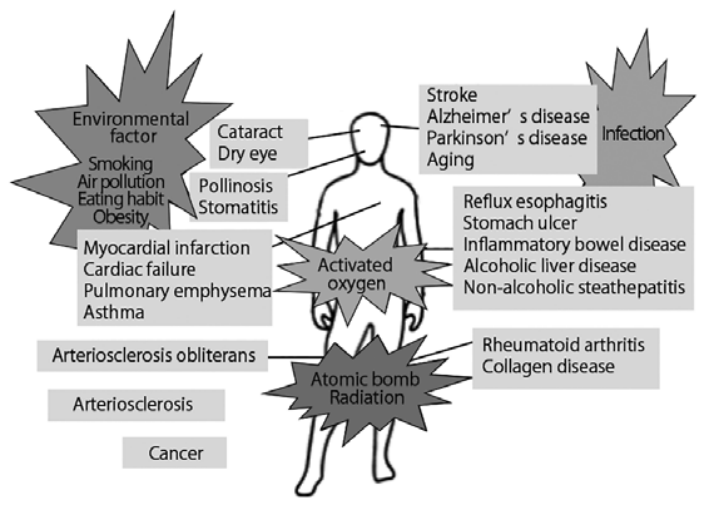

Figure 1 Activated oxygen and disease

\section{Understanding the Formidability of Living Organisms}

Since March 11, 2011, researchers, especially physicists, have begun to ascribe to the theory that "If the double-stranded of DNA (gene) is cut, it is permanently damaged; this damage accumulates, and cancer develops. I disagree with this notion and believe that it reflects a lack of understanding of the recent discoveries in biology. The fact is that cancer does not develop so easily or rapidly. In fact, even if there is some damage to double-strand chain DNAs, the majority is usually repaired.

Even if a genetic mutation occurs, it does not immediately become a life-threatening form of cancer in living organisms. The first defense system is an antioxidant, glutathione, and an 
anti-oxidative enzyme that living organisms have. These detect and detoxify the oxygen radicals. Next, the system that immediately assesses and restores the mutation when the gene is damaged operates. A representative tumor-suppressor gene, p53, plays an important role by checking whether the damage is too severe to repair and allows the cell to self-destruct. The mutant cells that pass through these processes are targeted and eliminated by immune cells. This process clearly functions like a multi-stage cancer protection system. Note, that organisms that breathe oxygen and live on land have acquired this system to protect them from the damage caused by leaking oxygen, rather than a system that has been acquired specifically to guard against radiation.

Biologists believe organisms can live on Earth because they have acquired this multi-protection system through an evolutionary process in which a dangerous substance must be removed; a gene must be repaired, if damaged; a cell must die if it cannot be repaired; and the same cell must be destroyed if none of the above works (Figure 2). The important principle for everyone to understand is that oxygen-breathing organisms are routinely damaged by reactive oxygen; therefore, human beings could not survive for 80 years if they could not self-repair after exposure to low dose radiation. After the Daiichi Accident, many medical doctors were reported in the media as saying that smoking has a higher cancer risk than exposure to radiation at the levels found in Fukushima. From my point of view, this was obviously true; however, physicists expressed their opposition to the notion, arguing that the effects of radiation and the effects of smoking cannot be compared. On the contrary, these effects are comparable. The effect of low-dose radiation is largely due to the reactive oxygen produced when radiation hits water, and smoking and various mutations can also generate oxygen radicals.

In regard to the evolution of the earth, there was no free oxygen during the initial stage of the birth of life. Oxygen became available with the emergence of photosynthetic organisms, and finally, oxygen-breathing organisms evolved. Since breathing oxygen is accompanied by oxidative damage, oxygen-breathing organisms also evolved and developed a system to overcome damage from oxygen radicals. Without this basic understanding that it is not necessarily catastrophic when a gene or cell is damaged, fear surrounding low-dose radiation cannot be overcome. Living organisms are repeatedly undergoing cycles of injury and repair.

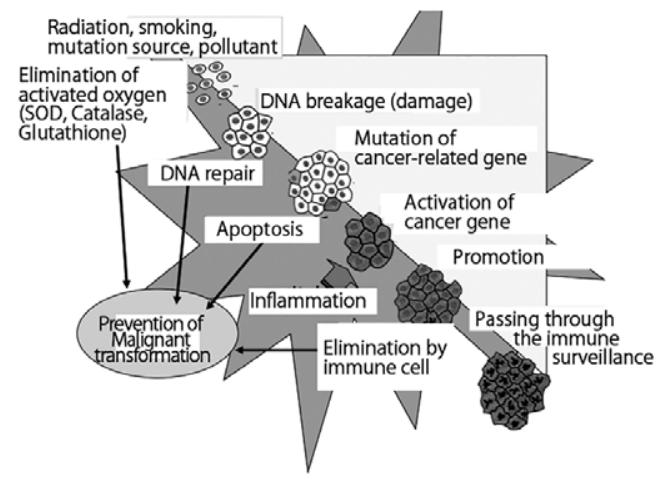

Figure 2 Malignant transformation process and multi-stage suppression mechanism

\section{Effects of Radiation and Ability to Defend}

It is generally believed that our body's response to radiation depends on the level of exposure. Specifically, the probability of an effect increases with dose. The effects of high dose 
radiation that exceeds a certain threshold are called deterministic; everyone exposed at these high levels will show the same negative symptoms. However, the effect that may occur as a result of low doses of radiation are considered probabilistic; meaning negative effects don't always occur, and when they do, the severity of these radiation-induced effects are different in each individual.

While this is an accepted theory, I do not believe these definitions should be used in health education. There are people who develop cancer and those who do not even though they are exposed to the same levels of radiation. Thus, it is also a fact that the outcome of radiation exposure cannot always be determined by probability. In reality, the balance among the amount of mutation source, the quality of the host defense system, the genetic background as well as the life style of the individual are very important factors that determine the effect of low dose radiation. Due to this, it is important to undertake a lifestyle that increases our natural defensive abilities. The way an individual lives from now on will change his or her life 20 or 30 years in the future.

When considering an appropriate lifestyle to improve immune ability, it should be noted that fear and stress worsen immune function, while a life lived with purpose and laughter improves it. In the previous studies, the activity of natural killer cells was enhanced in participants who were given cosmetic therapy. The same effect was found for image therapy, in which people imagined that their immune cells were attacking cancer cells. As an application of the cosmetic therapy, Fukushima residents who attended my lectures were given hand massages. The hand massage is warming and improves blood flow to the hands. Additionally, although these results are only preliminary, decreased amylase activity was observed in participants after the massage, indicating that stress was decreased. Above all, participants' mood softened. It seems that through these lectures, participants learned first-hand how they can better deal with the effects of low dose radiation by improving their immune system, rather than worrying a lot.

At this point (three and a half years since the accident), it is clear that the initial concern radiation contaminated food was unwarranted. This is evident from the results of the wholebody counter tests in residents and the testing of meals prepared by households in Fukushima by Co-op Fukushima (one extra meal was prepared for two days and sent to an inspection center to precisely measure the radiation dosage). I am convinced that there will be no significant health consequences due to low dose radiation in children in the near future if children are eating well. I believe there is a need for more people to eat meals that are high in antioxidants, so as to overcome any damage caused by radiation thus far, and as a way to prevent cancer and adult diseases in the future.

Instead of radiation, a more realistic problem in Fukushima in the near future is obesity in children and evacuees, which is a result of stress and lack of exercise.

\section{Moving Beyond Scientific Issues and the Effects of Low- Dose Radiation}

\section{Effects of Radiation and Crisis Communication}

It was shocking to see the graphical representation of evacuees moving out of Fukushima Prefecture. Since timely data on the evacuees were not compiled in March 2011, the actual situation immediately after the accident is unknown. However, it is apparent that the number 
of residents moving outside the prefecture increased around the summer of 2011 until early 2012 (Figure 3). The number of evacuees who moved to neighboring Yamagata Prefecture was particularly astonishing.

Looking back, the situation regarding the Fukushima Daiichi Nuclear Power Plant should have calmed down to a certain degree by the summer of 2011. It seems that the tense atmosphere that remained was affected by: (1) the comments by cabinet advisor Toshiso Kosako on April 29, 2011 that the radiation reference value of $20 \mathrm{mSv} / \mathrm{year}$ on a school ground was unacceptable and, (2) the testimony of Tatsuhiko Kodama, a professor at The University of Tokyo, at the Diet on July 27, 2011. Both had a big effect on the situation. However, when the author interviewed residents who evacuated not immediately after, but some time after the accident, they said that they decided to evacuate because they were skeptical about the government's statements when the decision was made to evacuate Iitate village one month after the accident. Many evacuees believed they could no longer trust the government because of the confusion caused when the evacuation order was issued one month after the accident, although just two weeks prior the government said that there was no immediate need to evacuate Iitate village. The reason why such an overwhelming number of Fukushima residents choose to evacuate out of the prefecture should be examined thoroughly in the future.

In addition, the effects of the dispute among scientists over the effects of low-dose radiation cannot be ignored. I noted that after the accident, researchers in the field of physics and biology/medicine who spoke out in the media had different perceptions of radiation from researchers in physics and biology/medicine in Kyoto. In times of crisis, it may be better to have a thorough interdisciplinary discussion among scientists to address differences in opinion and reach a consensus before speaking to the public.

This time, there were significantly different notions of "radiation" and "gene damage," especially among the researchers in the fields of physics and medicine/biology which seemed to cause confusion. The ALARA principle (As Low As Reasonably Achievable) has taken root among physicists, and there were strong sentiments against even the tiniest amount of radiation exposure that minimally exceeded the norm. On the other hand, researchers in the fields of medicine and biology understand that extremely high doses of radiation are used beneficially in cancer therapy and that genes are repeatedly damaged and repaired enabling humans to survive.

Three and a half years after the accident, the problems caused by excessive evacuation have also become clearer. Being over-cautious against every risk was the source of major confusion at the time of the crisis. Unlike short-term evacuation, the negative effects of long-term

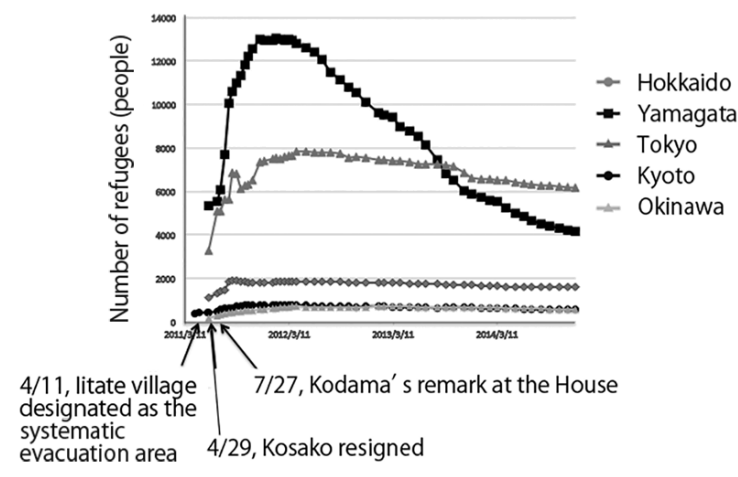

Figure 3 Movement of refugees from Fukushima to outside prefecture 
evacuation are blatant and cannot be ignored. In particular, the evacuation order based on the distance from the Fukushima Nuclear Power Plant brought miserable consequences to so-called "Weak evacuees", such as sick and elderly residents ${ }^{6}$. Currently the Japanese Red Cross Society is reviewing their guidelines for nuclear emergencies. Unlike other disasters, the protocol for nuclear disasters should be that residents remain where they are and evacuate only after deciding where to go; this will minimize the number of evacuees. Although countermeasures were implemented such as installing a filter unit to reduce the radiation leak from the nuclear power plant (based on the lessons learned from the Three Mile Island and Chernobyl disasters), what should be reflected upon is that there were no discussions about how to prepare for a nuclear emergency in Japan.

\section{Education about Radiation, Atomic Bombs, and Nuclear Power Generation}

Japan has experienced atomic bombs, and has stopped considering and arguing calmly about the effects of various doses of radiation. Having seen that atomic bombs and nuclear power generation were talked about with almost similar images on occasion, I felt a harmful influence in the past 40 years, during which we have not provided proper education about radiation. It is desirable to learn about the scientific side of radiation to limit education about radiation to the atomic bombs and nuclear disasters. The need to start with the commonplace, such as determining the dosage and scientifically evaluating it, will be increasingly called into question in the future.

\section{Issues That Cannot be Solved with Science}

The problem: the evacuation of Fukushima residents, which was meant to be only temporary, became prolonged and developed into a dire problem. The facts: houses that were intact with no major damage were located in 'no-go' areas and became breeding grounds for wild mice. These problems and facts weigh heavily on the hearts of the evacuees. In Minamisoma city, some residents commented that, "I do not grow the rice, if it cannot be sold. I have the compensation money anyway." Fukushima rice has very poor sales because it has developed a reputation for being unsafe. Food-service brokers took advantage of this, by demanding drastic price reductions, telling farmers, "The price difference would be compensated for with government reparations anyway." I realize that Fukushima residents have so many problems that cannot be solved by simply explaining low dose radiation and its scientific effects.

\section{References}

1) Uno K, Nakano K, Maruo $\mathrm{N}$ et al. The determination of IFN $(-\alpha)$ producing capacity in patients with various diseases and healthy persons using whole-blood cultures. J. IFN Cytokine Res. 1996; 16:911-918.

2) Uno K, Setoguchi J, Tanigawa $M$ et al. Differential interleukin 12 responsiveness for interferon gamma production in advanced stages of cancer patients correlates with performance status. Clin Cancer Res. 1998; 4:2425-2432.

3) Uno K. Anti-aging of immunity. Sogo-Rinsho (Clinic All-Round.) 2011;60:343-350. [in Japanese]

4) Kusunoki Y, Hayashi T. Long-lasting alterations of the immune system by ionizing radiation exposure: implications for disease development among atomic bomb survivors. Int. J. Radiat. Biol. 2008; $84: 1-14$.

5) Ozasa K, Shimizu Y, Suyama A, et al. Studies of the mortality of atomic bomb survivors, Report 14, 1950-2003: an overview of cancer and noncancer diseases. Radiat Res. 2012; 177:229-243.

6) Aikawa Y. Weak evacuee: Toyo Keizai Inc; 2013. [in Japanese] 